\title{
BMJ Global Health Learning from changes concurrent with implementing a complex and dynamic intervention to improve urban maternal and perinatal health in Dar es Salaam, Tanzania, 2011-2019
}

\author{
Brenda Sequeira Dmello (D) , ${ }^{1,2,3}$ Ziada Sellah, ${ }^{4}$ Grace Magembe, ${ }^{4}$ \\ Natasha Housseine (iD , ${ }^{3}$ Nanna Maaløe, ${ }^{2,5}$ Thomas van den Akker, ${ }^{6,7}$ \\ Dan W Meyrowitsch, ${ }^{2}$ Timothy Mushi, ${ }^{1}$ Mangalu Masweko, ${ }^{1}$ Dorcas Jidayi, ${ }^{1}$ \\ Jos van Roosmalen, ${ }^{6,7}$ Hussein L Kidanto ${ }^{3}$
}

To cite: Sequeira Dmello B, Sellah Z, Magembe G, et al. Learning from changes concurrent with implementing a complex and dynamic intervention to improve urban maternal and perinatal health in Dar es Salaam, Tanzania, 2011-2019. BMJ Global Health 2021;6:e004022. doi:10.1136/ bmjgh-2020-004022

Handling editor Valery Ridde

- Additional material is published online only. To view, please visit the journal online (http://dx.doi.org/10.1136/ bmjgh-2020-004022).

Received 28 September 2020 Revised 24 November 2020 Accepted 26 December 2020

Check for updates

(c) Author(s) (or their employer(s)) 2021. Re-use permitted under CC BY-NC. No commercial re-use. See rights and permissions. Published by BMJ.

For numbered affiliations see end of article.

Correspondence to Dr Brenda Sequeira Dmello; bseqdmello@gmail.com

\section{ABSTRACT}

Introduction Rapid urbanisation in Dar es Salaam, the main commercial hub in Tanzania, has resulted in congested health facilities, poor quality care, and unacceptably high facility-based maternal and perinatal mortality. Using a participatory approach, the Dar es Salaam regional government in partnership with a nongovernmental organisation, Comprehensive Community Based Rehabilitation in Tanzania, implemented a complex, dynamic intervention to improve the quality of care and survival during pregnancy and childbirth. The intervention was rolled out in 22 public health facilities, accounting for $60 \%$ of the city's facility births.

Methods Multiple intervention components addressed gaps across the maternal and perinatal continuum of care (training, infrastructure, routine data quality strengthening and utilisation). Quality of care was measured with the Standards-Based Management and Recognition tool. Temporal trends from 2011 to 2019 in routinely collected, high-quality data on facility utilisation and facility-based maternal and perinatal mortality were analysed.

Results Significant improvements were observed in the 22 health facilities: $41 \%$ decongestion in the three most overcrowded hospitals and comparable increase in use of lower level facilities, sixfold increase in quality of care, and overall reductions in facility-based maternal mortality ratio $(47 \%)$ and stillbirth rate $(19 \%)$

Conclusions This collaborative, multipartner, multilevel real-world implementation, led by the local government, leveraged structures in place to strengthen the urban health system and was sustained through a decade. As depicted in the theory of change, it is highly plausible that this complex intervention with the mediators and confounders contributed to improved distribution of workload, quality of maternity care and survival at birth.

\section{INTRODUCTION}

Despite increasing numbers of facility births, maternal and perinatal outcomes remain

\section{Key questions}

What is already known?

- In low-income and lower-middle-income countries a tendency to higher maternal and child mortality rates in urban versus rural areas, despite high rates of facility births in urban settlements, calls for context-specific implementation research and service delivery redesign to provide acceptable quality care in overcrowded, low-resource maternity units.

What are the new findings?

- Set in the rapidly growing urban city of Dar es Salaam with a population of six million people, a complex maternal and perinatal intervention cocreated by a public-private partnership resulted in multiple improvements in 22 strategically selected public health facilities, accounting for $60 \%$ of facility births in the city.

- Analysis of temporal trends from the intervention's embedded strengthened routine data (2011-2019) revealed significant decongestion in the three most overcrowded hospitals and comparable increase in use of lower level facilities, sixfold increase in quality of care, and concurrent overall reductions by $47 \%$ and $19 \%$ in facility-based maternal mortality ratio and stillbirth rate.

What do the new findings imply?

- Effective implementation of established best practices in real-world settings to reduce maternal and perinatal morbidity and mortality requires local leadership, collaborative partnerships, and an integrated, context-tailored health systems approach addressing the continuum of care, with a focus on strengthening surveillance and response systems.

poor in sub-Saharan Africa (sSA). ${ }^{12}$ Almost two-thirds of the 295000 global maternal deaths and the 2.5 million neonatal deaths in 
2017 occurred in sSA. ${ }^{34}$ In what may appear to be a positive momentum of high rates of facility births, massive urbanisation leads to geographically highly concentrated low-income urban communities and overburdening of under-resourced urban health systems. This paradoxically causes an 'urban disadvantage' for women giving birth. ${ }^{2} 56$ Tanzania is an evident example. The East African country's urban population has increased from 6\% in 1967 to an estimated $34 \%$ in 2018, concentrated primarily in the rapidly growing city of Dar es Salaam (DSM). ${ }^{5}$ Compared with rural areas, lower life expectancy and higher maternal and child mortality in urban areas raise concerns, and information on contributing factors and effective interventions are warranted. ${ }^{125}$

In 2009, the burden of urbanisation on maternity and newborn care was already apparent in DSM. ${ }^{7-10}$ Of the 69008 annual births in the DSM region, 90\% occurred in three public health facilities (HFs) where there was a lack of human resources with proficient maternity and neonatal skills, combined with a non-conducive infrastructure and lack of essential equipment and supplies. ${ }^{711}$ Congestion was most severe in the city's three municipal hospitals, where annual childbirth ranged from 16000 to 23000 . At the same time, primary level HFs were underutilised. ${ }^{7811}$ With $90 \%$ of all births in the city occurring in HFs, the facility-based maternal mortality ratio of 159-218 per 100000 live births and the perinatal mortality rate of 27-44 per 1000 births were alarmingly high. $^{711}$

To increase maternal and perinatal newborn survival during birth in DSM, a public-private partnership was established in 2010 between the regional health authorities and the non-governmental organisation (NGO), 'Comprehensive Community Based Rehabilitation in
Tanzania' (CCBRT). The following 10 years of joint efforts aimed to improve quality of care and survival during birth at all levels of the health system both directly by clinical training and supervision and indirectly through associated improvements in health system infrastructure and data quality and utilisation. As an intermediate step in enabling health providers to provide best possible care, it was hypothesised that a strengthened referral system and more skills and resources at primary HFs would cause a safe redistribution of workload from the congested maternity hospitals to underutilised HFs.

Drawing from the Standards for Reporting Implementation Studies (StaRI), this paper presents the implementation strategy and intervention components (2010-2019) and concurrent changes in distribution of births, quality of care and birth outcomes. ${ }^{12}$

\section{METHODS}

\section{Setting and inclusion of HFs}

DSM is one of the fastest growing cities in sSA. With a population approximating six million in 2019 , it is expected to become a megacity with over ten million inhabitants by $2030 .^{13}$ An increasing ratio of births over deaths, migration and administrative reclassification of city boundaries all contribute to this increase. ${ }^{2}$ DSM scores lowest among African cities on the Urban Health Index, which includes indicators of access to water and sanitation, use of solid fuels, women's education, women's knowledge of HIV and child healthcare coverage. ${ }^{2}$ While most wealth in Tanzania is concentrated in cities, wealth inequality is higher in urban than in rural areas. ${ }^{2}$ Additional relevant outer and inner contextual factors are represented in figure $1 .^{251415}$

Figure 1 Contextual factors in Dar es Salaam (2010-2019) that may have influenced outcomes of implementation. CCBRT, Comprehensive Community Based Rehabilitation in Tanzania; CDC, Center for Disease Control; GoT, Government of Tanzania; Jhpiego, Johns Hopkins Program for International Education in Gynecology and Obstetrics; MDG, Millennium Development Goals; MDH, Management and Development for Health; NGO, non-governmental organisation; SDG, Sustainable Development Goals. 


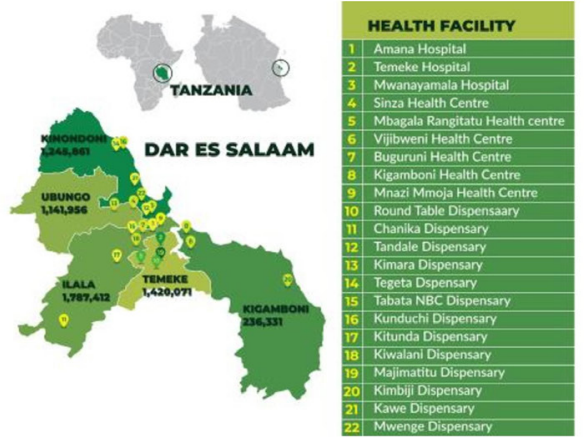

B

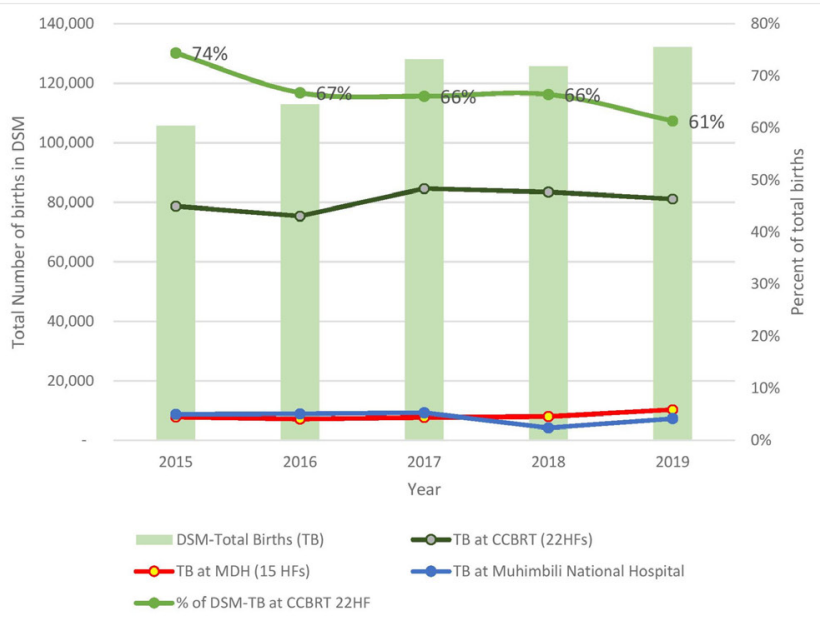

Figure 2 (A) Study setting: map of DSM region indicating the $22 \mathrm{HFs}$ in the 5 municipalities of Dar es Salaam. The intervention programme's phase 1 (2010) included facilities numbered 1-8, phase 2 (2011) included facilities numbered 9-16, and 17-22 were added in phase 3 (2013). In 2010, the region had three municipalities (Ilala, Kinondoni and Temeke), but in 2016 Kigamboni was carved out of Temeke municipality and Ubungo subdivided from Kinondoni. Population estimates derived from Tanzania's National Bureau of Statistics' 2019 projections. (B) Total births (TB) in Dar es Salaam and Total Births in the 22 health facilities supported by the urban maternal and newborn healthcare intervention (2010-2019) (data source: DHIS2). MDH supported 15 HFs. The remainder of births ( 10\%) occur in private institutions and other public dispensaries. CCBRT, Comprehensive Community Based Rehabilitation in Tanzania; DSM, Dar es Salaam; HF, health facility; MDH, Management and Development for Health.

With more than $90 \%$ of births in DSM occurring in public facilities, this maternal and newborn intervention focused on strengthening the public maternity care system. ${ }^{11} 1617$ The $22 \mathrm{HFs}$ were enrolled into the intervention in three phases. The initial phase started in 2010 and consisted of support to eight HFs (three high-volume hospitals and five health centres), identified in the baseline assessment. ${ }^{11}$ As additional funding was secured, the programme expanded in two additional phases (2011, 2013 ) to support a total of $22 \mathrm{HFs}$ (figure 2A), which collectively assisted more than $60 \%$ of all facility births in DSM during the 10 years studied (figure 2B).

\section{Intervention design and strategy}

The programme for strengthening maternal and newborn care in DSM was co-designed by the DSM regional health authorities and CCBRT, with additional contributions from multiple partners (online supplemental file 1). The specific components of the complex health system intervention were selected in accordance with the needs revealed in a baseline assessment in 8 of the $22 \mathrm{HFs},{ }^{11}$ and formulated as a theory of change (figure 3 ). ${ }^{18}$

The theory of change was that some of the positive changes in quality of care and survival during birth would be directly related to strengthened competencies of health providers (clinical training, introducing a standards-based approach to care and supervision) and stable access to essential equipment, medicine, emergency surgery and blood transfusion. Other parts of change would be indirectly stimulated through improved data quality and utilisation (audits and quality improvement meetings) and decongestion of the overcrowded municipal hospitals (improved referral system with redistribution of births to upgraded primary level HFs).

Evidence-based best practices for each intervention component were extracted and adapted from the national strategic plans and reviews of international scientific literature. ${ }^{190}$ The intervention was adjusted over time in response to successes and challenges, with new elements systematically layered on as new gaps emerged. The core components of the complex intervention are described here, and more details are available in box 1 and online supplemental file 2 .

Regional leadership of the multistakeholder collaboration and care coordination

The regional health leadership ensured alignment to national strategic priorities, guided the implementation approach, designated focal persons at regional and municipal levels to support coordination, and led the quarterly improvement meetings. In addition, an initial multistakeholder meeting enabled mapping of partners working in maternal health, further enabling collaboration and synergy. This strengthened the system-level coordination, which was crucial for jointly developing referral criteria for women with high-risk pregnancies, easily allowing inputs from health providers and managers across the HFs. Thereby, timely recognition of clinical risks, rapid resuscitation and referral of women who developed obstetric emergencies were promoted, from primary care HFs to hospitals within the referral network. The 22 HFs were linked through a closed user group, with a phone call to follow up on outcomes of referred women.

Selection of the HFs targeted government-owned HFs, with high utilisation and/or those planned for upgrades. Following initial experiences during implementation in 2010 and 2011, the regional authorities led the scale-up to an additional $21 \mathrm{HFs}$ (6 dispensaries supported by CCBRT and 15 dispensaries supported by another NGO, Management and Development for Health). 


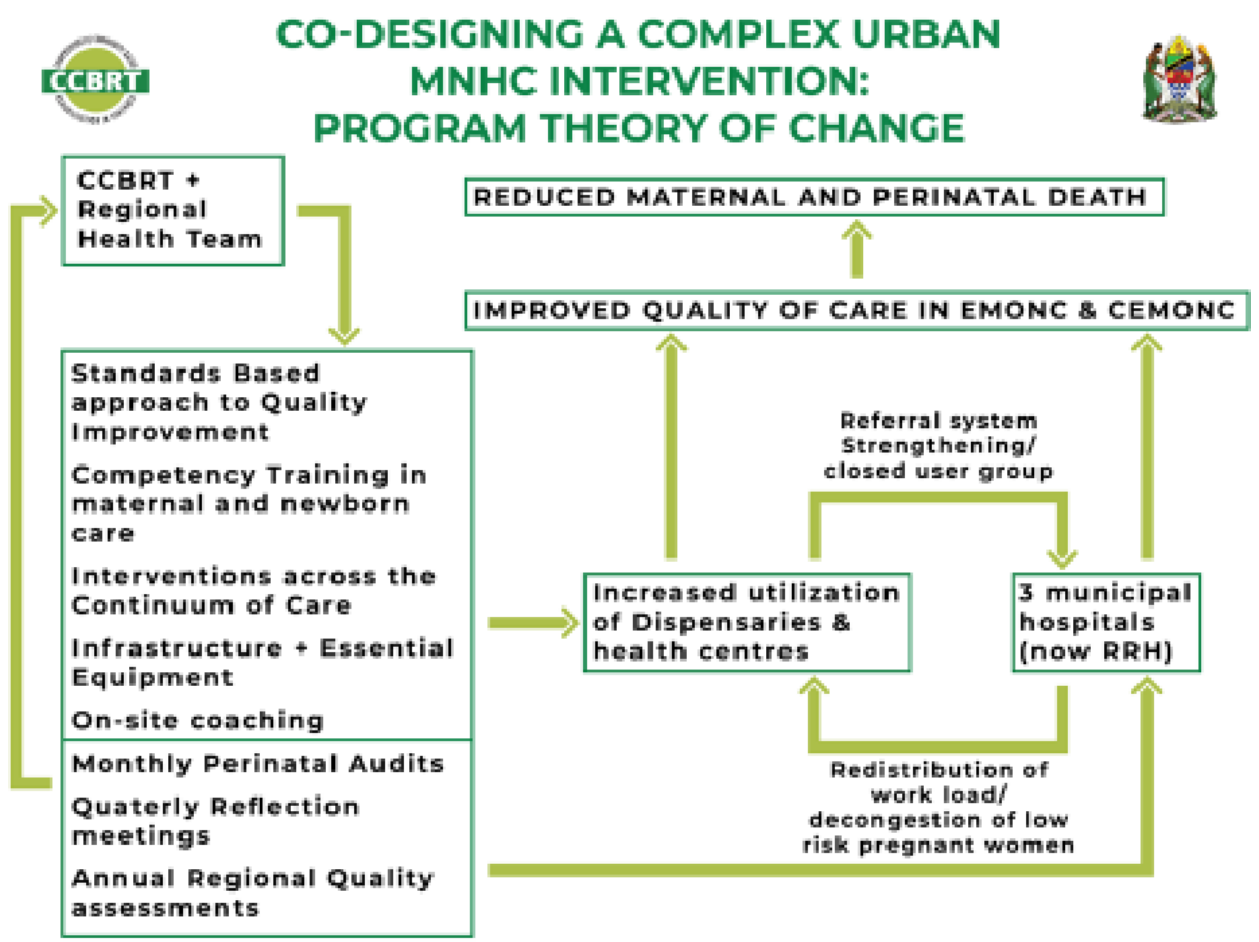

Figure 3 Theory of change for the complex urban maternal and perinatal healthcare intervention in Tanzania: implementation strategy and interventions. CCBRT, Comprehensive Community Based Rehabilitation in Tanzania; CEmONC, Comprehensive Emergency Obstetric and Neonatal Care; EmONC, Emergency Obstetric and Neonatal Care; RRH, regional referral hospitals.

Standards-based quality improvement

The Standards-Based Management and Recognition approach (SBM-R), a validated performance and quality improvement method, developed by Jhpiego, was implemented collaboratively. ${ }^{21-23}$ SBM-R quality assessments in each facility were conducted by a team led by the regional nursing officer and consisting of representatives from the regional and municipal health offices, the HFs and CCBRT. Assessors spent 2-3 days in each facility performing direct observations and scoring (expressed in percentages) using the SBM-R tool and provided immediate feedback to the HFs. ${ }^{21-23}$

Improving essential clinical knowledge and skills

Intervention components were derived from the Tanzanian Reproductive, Maternal and Newborn Health roadmap and included interventions known to avert maternal and perinatal deaths across the continuum of pregnancy and childbirth. ${ }^{19}{ }^{20}$ Initial training targeted skills in routine intrapartum care and Basic Emergency Obstetric and Newborn Care (BEmONC). As gaps emerged, additional short trainings were added (antenatal care, essential newborn care, postnatal care, neonatal resuscitation, surgical skills, safe anaesthesia, kangaroo care, care for sick newborns, Comprehensive Emergency Obstetric and Newborn Care, referral recognition and management, and data quality and perinatal audits). The focus was on building the competencies of inservice healthcare providers. (online supplemental file $3)$.
Five training modalities were implemented: (1) 2-week BEmONC national training, facilitated by national trainers; (2) 5-day critical BEmONC skills course; (3) 1-day modular course on specific topics addressing gaps detected in perinatal audits; (4) on-site trainer: an experienced nurse or doctor supported by CCBRT was periodically stationed full time for periods from 2 weeks to 1 year in the labour ward of high-volume facilities for on-site coaching and support; and (5) on-the-job coaching (mentors would spend 2-3 days in each of the 22 sites, building skills during routine service provision).

Optimising infrastructure, equipment and essential medicines

Extensive investments were made to create a conducive environment for care through improved infrastructure: building operating theatres, improving flows and privacy, as well as gap filling of essential equipment and supplies. Access to safe provision of blood, anaesthesia and surgery was improved. Initial support was through donor-funded NGOs; however, from 2016 it was supported by the Ministry of Health and Social Welfare through the local government (online supplemental file 4).

Improving data quality and utilisation for continuous improvement Strengthening recording, reporting and utilisation of routinely collected facility data was a significant part of the intervention, which included support for printing new Health Management Information tools (HMIS), also called MTUHA (Mfumo wa Taarifa za Uendeshaji Huduma za Afya) in Swahili, as well as facility quality assurance extraction sheets, labour case notes and 
Box 1 Overview of the key components constituting the co-created, complex urban maternal and newborn healthcare intervention

\section{Organisational intervention components:}

- A participatory approach engaging regional and municipal authorities, planning needs assessment, and selection of interventions, tools and HFs (2009-2019).

- CCBRT provided technical oversight, supported advocacy, cocreation, resource mobilisation, harnessed collaborations with other stakeholders, and provided management and competency building (2009-2019).

- Partnerships mapping with international and local organisations for synergy (online supplemental file 1).

- Training of managers at all levels on the SBM-R quality improvement tool (2010).

- Regionally led, quarterly quality improvement meetings provided opportunities to adjust the intervention and address barriers (2012-2019).

- Capacity building of BEmONC national trainers, BEmONC focal persons (2012), facility nurses and doctors mentors (2013/2014/2015), and municipal and facility data focal persons.

On-the-job training with district facility mentors supported by CCBRT.

- Referral support network was created to link all health facilities in terms of technical and administrative support through closed user group phone communication (2014).

\section{Direct clinical intervention components:}

- Knowledge and skills training on BEmONC (2010-2019), essential newborn care and neonatal resuscitation (2010), training on standards of care (2010), later followed by antenatal care (2011), safe caesarean section and vacuum extraction skills, kangaroo care (2012), safe anaesthesia (2013), care of sick newborns (2015), prolonged labour/birth asphyxia and sepsis prevention, visual estimation of blood loss and blood use in haemorrhage (2016/2017), and nurses' ultrasound training (2016).

- Perinatal Problem Identification Programme (2014).

\section{Cross-cutting system strengthening components:}

- Improved facility-based routine data collection, quality assurance and data utilisation at HF level for decision making (2011).

- Counting and auditing perinatal and maternal deaths with continuous improvement through action via monthly facility-based audit meetings (2014 -2019).

- Investment in satellite blood banks in collaboration with the National Blood Transfusion Services to increase access to safe blood and community blood donation campaigns (2015).

- Infrastructure upgrades (online supplemental file 4).

Note: The year refers to the year that particular component was first introduced into the programme. See online supplemental files $2-4$ for more details on frequency.

BEmONC, Basic Emergency Obstetric and Newborn Care; CCBRT, Comprehensive Community Based Rehabilitation in Tanzania; HFs, health facilities; SBM-R, Standards-Based Management and Recognition.

neonatal admission data collection tools, and training more than 292 staff on utilisation of the tools. Routine facility data were validated by direct counting from registers by district managers and CCBRT. This took effect in 2011. Key indicators were presented in regional quarterly improvement meetings and disseminated to all managers at all levels. Leadership and managers would reflect on key indicators which included quality of care scores (SBM-R), cases referred, routine data, and maternal and perinatal deaths from each of the $22 \mathrm{HFs}$. These meetings provided an opportunity for identification of deficiencies in clinical skills, developing training plans, following up on HF action plans, improving peer accountability and dynamic adaptation of the programme (figure 4). The process was subsequently used for scale-up of the intervention to additional HFs.

\section{Evaluation design}

This was a retrospective analysis of a real-world complex intervention. The Donabedian model for analysing quality of care was applied to verify the programme's theory of change (figure 3), in that the complex intervention may have contributed to improved distribution of births (structure), quality of care (process) and survival (outcome).

\section{Structure and outcome data}

Data sources were the 22 HFs' HMIS from 2011 and onwards. Each month, key birth data were extracted from each HF. Temporal changes in facility births were used to determine redistribution of facility births over time. Total numbers of (live) births, mode of birth, and maternal and perinatal deaths were extracted from each HF. Facility maternal mortality ratios over time included maternal deaths from all causes per 100000 live births. Fresh and macerated stillbirths were calculated together due to misclassification of fresh stillbirths as macerated in some cases. Total early neonatal deaths that occurred in the HF were counted as well; these included neonatal deaths among inborn newborns and those deaths among sick newborns referred after birth elsewhere.

\section{Process data}

Quality of care was measured using the SBM-R tool in each HF through direct structured observations. Annual SBM-R results from 2010 to 2019 were transcribed from hard copy into an Excel sheet, and the average quality scores of all thematic areas resulted in overall quality scores for each HF, expressed as a percentage. While both antenatal care and BEmONC were measured, for this paper only SBM-R scores for BEmONC are presented. In addition, the number of staff receiving competency training was assessed.

Possible confounders and mediators include staffing, staff transition, workload changes, health budget, other clinical training interventions and population changes in DSM. Figure 1 presents some contextual factors. However, time and resources did not allow for detailed analysis of these variables.

\section{Patient and public involvement}

The study was designed based on a needs assessment done pre-implementation in $2009,{ }^{11}$ with the aim to improve service delivery, improve quality of care, and reduce 


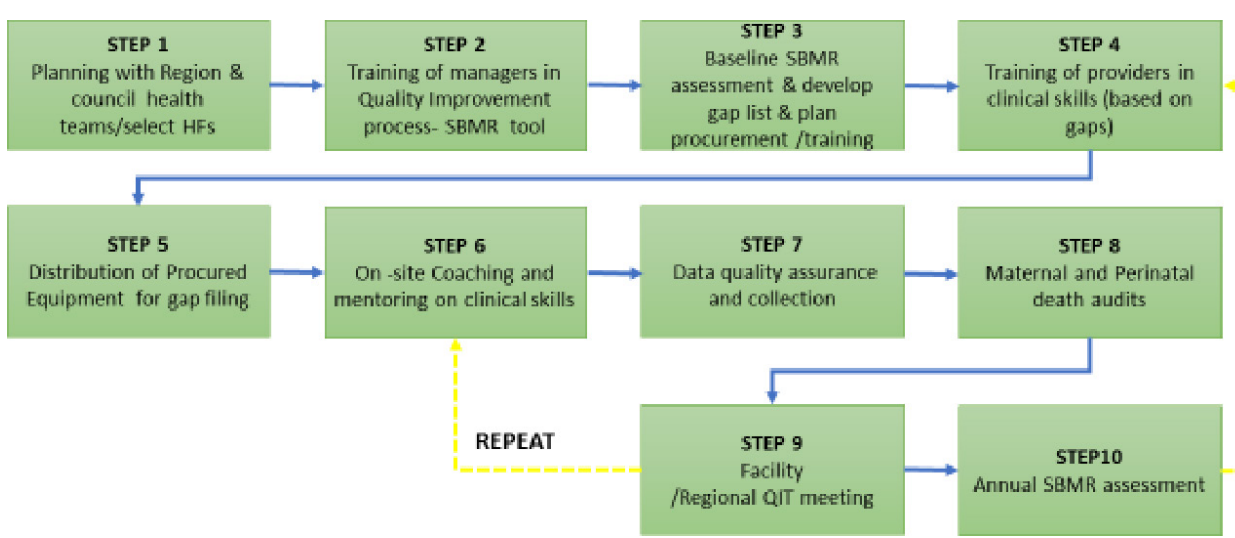

Figure 4 Scale-up and dynamic adaptation process with feedback loop for continuous improvement. HF, health facility; SBM-R, Standards-Based Management and Recognition.

poor maternal and perinatal outcomes. This included patient interviews (not included in this paper). During implementation, community health workers, community leaders and HF governing boards were among the stakeholders engaged and orientated on the programme, the routine outcome data and the intervention, and as end users of the supported HFs provided patient-level perspectives (online supplemental file 3). Data on

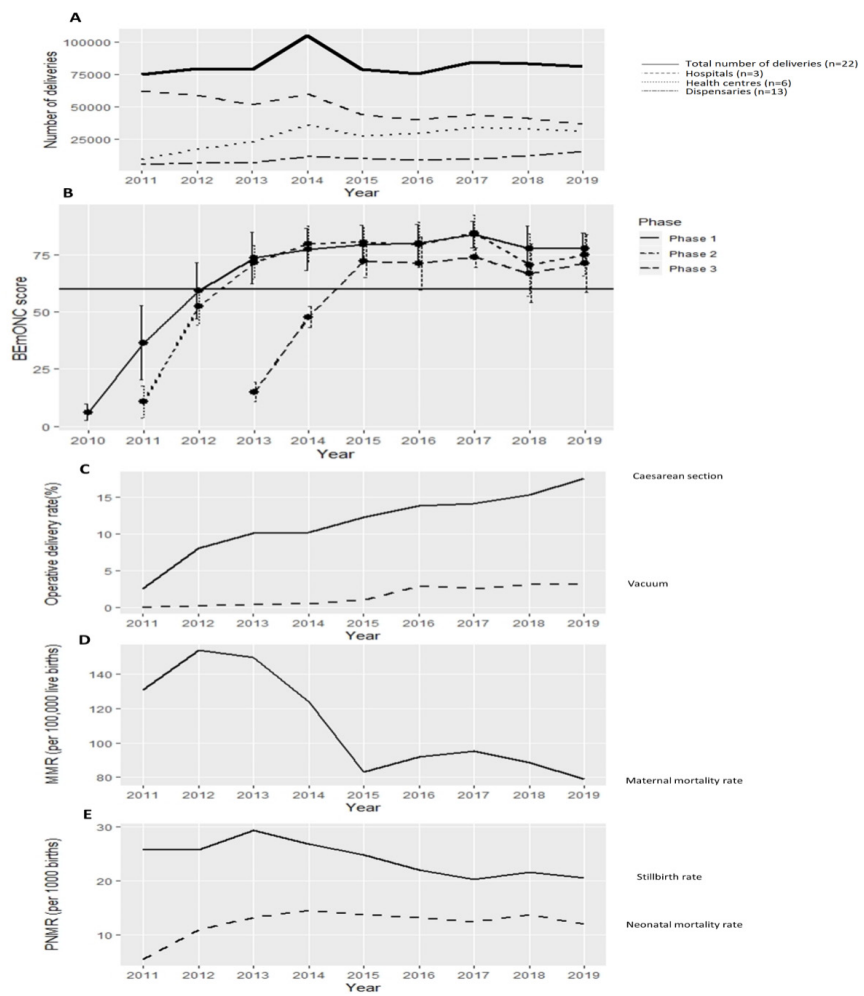

Figure 5 Associated changes through the co-created, complex urban maternal and perinatal health care intervention (2010-2019): redistribution of births (A), SBM-R quality score $(B)$, operative birth $(C)$, facility-based MMR (D), and stillbirths and neonatal mortality rate (E). The strong horizontal line in (B) represents the $60 \%$ bar that is the minimum standard performance expected for health facilities by the Ministry of Health. BEmONC, Basic Emergency Obstetric and Newborn Care; MMR, maternal mortality ratio; PNMR, Perinatal Mortality Rate. the quality of care, maternal and perinatal audits were reviewed by health managers in monthly meetings and by district and regional managers in quarterly meetings to ensure safety of women. Data used for analysis in this paper were routine, anonymised facility data.

\section{Statistical analysis}

Simple descriptive analyses were conducted using frequencies and percentages. Univariate generalised linear models for Poisson distributions were performed to detect changes over time in SBM-R scores, operative births, and the number of births, stillbirths, and maternal and neonatal deaths. SBM-R scores were analysed according to the phase each hospital first entered the intervention. The number of births was analysed according to the level of facility designated at the beginning of the intervention (hospitals $n=3$, health centres $n=6$, dispensaries $n=13$ ). Operative births and birth outcomes were analysed across all HFs. Results were reported as rate ratio (RR) with $95 \%$ CI, with RR representing the change in rate per year. Analyses were performed in Microsoft Excel (V.2013) and R V.3.5.3 (The R Foundation for Statistical Computing, 2019). ${ }^{24}$

\section{RESULTS}

From 2011 to 2019, a total of 741282 mother-baby pairs (online supplemental file 5) were assisted during childbirth in the 22 CCBRT-supported HFs. Collectively, these HFs supported between $61 \%$ and $74 \%$ of the total HF births in DSM between 2015 and 2019 (figure 2B).

\section{Structure: redistribution of births}

Redistribution of births was recorded from 2011 to 2019, with an average of $41 \%$ reduction recorded in the three overcrowded municipal hospitals (RR $0.93 ; 95 \%$ CI 0.93 to 0.94$)$ There was a concomitant $144 \%$ increase in utilisation in 6 upgraded health centres (RR 1.10; 95\% CI 1.09 to 1.10 ) and $117 \%$ increase in utilisation in 13 dispensaries (RR 1.10; 95\% CI 1.00 to 1.12) (figure 5A). Temporal changes in the total number of childbirths per HF are presented in online supplemental file 5 . 


\section{Process: quality of care}

In total, 7930 participants (mainly health care workers providing maternal and newborn health care services) were trained over the decade, in a variety of short and long courses and different topics to enhance competencies in caring for women and newborns across the continuum of care as well as skills to provide respectful care for pregnant women with disabilities. Participants included health managers $(\mathrm{n}=280)$ and community health workers and community leaders $(\mathrm{n}=788)$. Among the trainees, $1461(18 \%)$ received repeated sessions (online supplemental file 3 for details).

Quality of care, measured using the SBM-R tool, across all 22 HFs improved from a baseline average of $10.6 \%$ to $74.6 \%$ (RR 1.10; 95\% CI 1.09 to 1.11) (figure 5B). SBM-R scores for phase 1 HFs increased from $6 \%$ to $78 \%$ (RR $1.11 ; 95 \%$ CI 1.11 to 1.12 ); phase 2 HFs from $11 \%$ to $75 \%$ (RR 1.09; $95 \%$ CI 1.08 to 1.10 ); and phase 3 from $15 \%$ to $71 \%$ (RR: 1.13 ; $95 \%$ CI 1.11 to 1.16 ).

\section{Mode of birth}

Overall rate of caesarean section increased from $2.3 \%$ to $17.6 \%$ (RR 1.15; 95\% CI 1.14 to 1.15 ) and rate of assisted vaginal birth from $0.03 \%$ to $3.1 \%$ (RR $1.43 ; 95 \%$ CI 1.41 to 1.43 ) (figure $5 \mathrm{C}$ ).

\section{Outcome: maternal and perinatal survival}

During the decade, facility-based maternal mortality ratio reduced from 154 to 79 per 100000 live births (RR 0.92; $95 \%$ CI 0.9 to 0.95 ) (figure 5D), and stillbirth rate reduced from 26 to 21 per 1000 live births (RR 0.96; $95 \%$ CI 0.96 to 0.97 ). Neonatal deaths included the sum of deaths among inborn and referred babies and increased from 5.5 in 2011 to a peak of 14.5 in 2014 and then slightly declined to 12.1 in 2019 (RR 1.05; $95 \%$ CI 1.04 to 1.05) (figure $5 \mathrm{E}$ ).

\section{DISCUSSION}

By a collaborative, multilevel, multiple stakeholder participatory approach, a complex context-specific intervention was implemented. It addressed gaps across the maternal and newborn continuum of care and was sustained for a decade in the rapidly urbanising region of DSM, Tanzania. Such health system implementation research, with embedded strengthening and use of routine data, is called for from real-world, low-resource settings struggling with the greatest burden of preventable lost lives during birth. ${ }^{25-28}$

The intervention targeted 22 strategically selected government-owned HFs, which accounted for more than $60 \%$ of all facility births in the city, whereby simultaneous and significant improvements were observed: $41 \%$ decongestion in the three overcrowded hospitals, with comparable increased use of lower level facilities, sixfold increase in quality of maternity care, and reductions by $47 \%$ and $19 \%$ in facility-based maternal mortality ratio and stillbirth rate. Overall, our findings align with the proposed theory of change, and when considering possible mediators and confounders it appears plausible that the components of this complex intervention contributed to the improvements. ${ }^{27} 2930$ The authors recognise that despite the favourable outcome, the observed positive trends over the decade need to be interpreted with caution as this was not a controlled trial.

\section{Plausible contributors to the intervention's success}

Individual components of this complex intervention were not innovative and mainly included already evidencebased practices, which are considered routine and even women's rights in well-resourced settings. ${ }^{29}{ }^{31}$ However, despite knowing what works, many resource-constrained settings struggle with the implementation of best practices as routine care. This was the situation in DSM in 2009. ${ }^{11}$ This complex intervention's innovation lies in the successful translation of knowledge to implementation and sustainability of these best practices at scale in a realworld, low-resource setting. Four plausible contributors in this success are discussed here.

First, this programme, which was led and owned by the local health authorities, included central steps of 'problem-driven iterative adaptation' to effectively change practice; the broad set of stakeholders nominated and defined problems and assisted in tailoring the package of flexible interventions, which targeted multiple levels of the health sector and were dynamically implemented and evaluated throughout the decade. This ensured viable, legitimate, relevant and supportable reforms. ${ }^{32-35}$

Second, multiple evidence-based interventions addressing gaps along the entire continuum of maternal and perinatal healthcare were adapted and included in the complex intervention, each of which has previously been proven effective elsewhere. ${ }^{19}{ }^{30}$ For instance, the introduction of standards-based clinical care, enabled through use of the SBM-R tool, proved to be an acceptable, scalable quality improvement method. Improved care in the $22 \mathrm{HFs}$ was similar to the effects seen in other regions of Tanzania, as well as in Ethiopia and Nigeria, where the SBM-R tool is widely used. ${ }^{21-23}$

Third, data improvement efforts were an embedded part of the intervention, as recommended by effective large-scale maternal health programmes in Africa. ${ }^{36}{ }^{37} \mathrm{In}$ particular, in synergy with the SBM-R approach, facilitybased analysis and utilisation of perinatal audit data in quality improvement meetings appeared central to understand the opportunities to avoid adverse events.

Fourth, strategic decongestion of the dangerously overcrowded urban hospitals through revitalising selected primary upgraded HFs resulted in redistribution of workload and improved provider-to-labouring women ratios in the most congested facilities. Notably, the overall volume of workload in the $22 \mathrm{HFs}$ remained unchanged over the last 5 years of the study, suggesting redistribution instead of decreasing HF birth rates (online supplemental file 5). The observed rapid increase in utilisation of the upgraded HFs, now providing comprehensive Emergency Obstetric and Neonatal Care (EmONC), is 
similar to what has been reported from other regions of Tanzania, ${ }^{38}{ }^{39}$ but the magnitude of increased workload is unprecedented in published literature; in 3 years, annual workloads increased from less than 3000 (range 329-2622) to above 10000 births (online supplemental file 5).$^{7811}$ Surprisingly, dispensaries that offered only basic EmONC also noted increased utilisation for birth, although far more gradually. During this extreme transformation, the level of patient safety remained stable, and even improved, through strengthened clinical competencies, regular support visits, review of the outcome indicators and the strengthened referral system. Hence, the data suggest that an integrated systematic sustained quality improvement approach is helpful to reduce the bypass phenomenon, thereby maximising functionality of the health system in low-resource urban settings. ${ }^{38} 40$

\section{Strengths and limitations}

The StaRI checklist was useful in structuring this evaluation, describing a real-world implementation, using routine data to generate trends over time. ${ }^{1230}$ Overall, the evaluation followed the principles of the emerging theory of contribution analysis (CA),${ }^{41}$ which is meant to answer "whether the intervention, in light of the multiple factors influencing a result, has made a noticeable contribution to an observed result and in what way?' CA is particularly useful in retrospective evaluations like the present study, which did not allow design of an experiment to evaluate impact. While the lack of adequate control facilities and the inherent challenges in evaluation of multifaceted complex intervention within a dynamic sociopolitical context are limitations of this study, ${ }^{18}$ by verifying the theory of change that the programme is based on and paying attention to other contextual factors that may have influenced outcomes (figure 1), our findings infer a plausible relationship between the intervention and the favourable outcomes. ${ }^{41} 42$

We recognise the potential roles of broader contextual confounders and mediators during a decade of intense global attention for maternal and newborn healthcare strengthening through the Millennium Development Goals and Sustainable Development Goals. ${ }^{36} 37$ Central confounders and mediators to the changes in quality of care and birth outcomes might include changes in staffing, health budget increases or cuts, introduction of other clinical training interventions, changes in the pregraduate curriculum and urban population changes. However, unsafe low staffing versus workload ratios unfortunately persisted in the city during the years studied, and neither did the health budget nor staffing improve, while urbanisation continued.

Other limitations of this evaluation include challenges with the SBM-R tool; it provides a cross-sectional measure of quality and fails to adequately measure clients' experience of care. ${ }^{43}$ Moreover, while this evaluation addressed a programme aimed at improving 'intra-hospital' quality of care and birth outcomes in the supported HFs, the analysis included prehospital stillbirths and mortality among sick neonates who were referred from home or non-supported facilities. This may explain increased neonatal mortality rates observed in the supported HFs that included three out of the four public neonatal high care units in DSM.

Programmatic challenges included frequent staff transfers, including leadership changes, with periodic skills attrition as new staff with substandard life-saving skills would be rotated into the maternity wards every couple of months. This could account for previous studies reporting poor effects of training and supervision on improving provider skills. ${ }^{44}$ Mitigation efforts, in this study, included frequent refresher and on-the-job training. Exploring the feasibility of building capacity of ward in-charges to conduct introductory training for new staff, exploring modalities for introducing low-dose, high-frequency on-the-job training, as well as investing in preservice education to improve health providers' competencies are recommended. ${ }^{45} 46$

\section{Implications for the future}

Despite decongestion, annual workloads remained above 10000 births in five HFs, a reflection of the urban challenge of high population increase and popularity of public HFs for childbirth. ${ }^{1617}$ Unsafe low staffing versus workload ratios persisted, similar to urban maternity units in other low-income and middle-income settings. ${ }^{46}$ Congested primary level hospitals present an inherent risk that women with low-risk and high-risk pregnancies compete for attention from the few available staff, creating an unconducive environment with potential intrafacility delay or even neglect in receiving care. ${ }^{47}$ Improved skills and infrastructure upgrades cannot adequately compensate for critical staff shortages in highintensity work areas like those experienced in maternity and neonatal units in urban low-resource settings. Unless addressed, unnecessary preventable deaths will continue to occur, irrespective of the complexity and sophistication of the interventions.

Increased utilisation of lower level centres without compensatory budget and sufficient allocation of essential drugs and human resources created a strain on primary care facilities. This required responsiveness of the public-private partnership to bridge funding gaps, while awaiting local government planning and budget cycle, when the initiatives were later absorbed into the HFs' budgets.

Moreover, the alarmingly low baseline quality of maternity care (average low SBM-R scores of $10.6 \%$ ) in this study requires consideration of whether non-upgraded HFs are safe for childbirth. Standard care during birth is an ethical priority for health managers to consider. Fortunately, improvement occurred rapidly following the SBM-R approach, more so in phases 2 and 3, as the scale-up process was refined (figures 4 and $5 \mathrm{~B}$ ).

Implementation strategies of this regionally led, public-private partnership to systematically strengthen the health system may have applicability in other similar 
low-resource settings affected by low-quality care and high maternal and perinatal mortality. Implementation research to explore the impact of the strategy and the package of interventions in other regions, preferably both urban and rural, would be useful to better determine 'attribution' of active ingredients of the programme on outcome.

\section{CONCLUSION}

This multilevel, multistakeholder, context-tailored package of maternal and newborn health interventions was feasible, acceptable and sustainable in a congested urban health system, and the simultaneous changes in quality of care and survival are encouraging. Interventions that have been evaluated as effective elsewhere were a starting point, but not the end point in getting what works to happen, and the programme was based on local, problem-driven adaptation and flexible implementation. ${ }^{48}$ The resulting comprehensive, contextspecific complex intervention included both "point of care interventions' and system-based components, highlighting the importance of high-quality health systems in reducing preventable deaths during birth. ${ }^{27} 2849$ Addressing residual gaps in the quality and quantity of human resources for health, particularly in high-volume urban birthing units, is urgently required.

\section{Author affiliations \\ ${ }^{1}$ Maternal and Newborn Health, Comprehensive Community Based Rehabilitation in Tanzania, Dar es Salaam, United Republic of Tanzania \\ ${ }^{2}$ Global Health Section, Department of Public Health, University of Copenhagen, Kobenhavn, Denmark \\ ${ }^{3}$ Medical College, Aga Khan University, Dar es Salaam, United Republic of Tanzania ${ }^{4}$ Director, Tanzania Ministry of Health, Community Development, Gender, Elderly and Children (MoHCDGEC), Dodoma, United Republic of Tanzania \\ ${ }^{5}$ Department of Obstetrics and Gynaecology, Hvidovre Hospital, Hvidovre, Denmark ${ }^{6}$ Athena Institute, Vrije Universiteit Amsterdam, Amsterdam, Noord-Holland, The Netherlands \\ ${ }^{7}$ Department of Obstetrics and Gynaecology, Leiden University Medical Center, Leiden, Zuid-Holland, The Netherlands}

Acknowledgements We would like to recognise the leadership of DSM regional and municipal health offices, the health facility management teams of the 22 CCBRT partner health facilities, the management of CCBRT, the CCBRT maternal and newborn healthcare team, and the healthcare providers in the $22 \mathrm{HFs}$ for accepting the interventions and their care for mothers and newborns in DSM. Special appreciation to Joel Missangu, who worked with BSD to develop figures 2 and 3, and Elizabeth Mrema for compiling the data on training (online supplemental file 3) and Sidney Mboya for support in developing online supplemental file $2 B$ and figure $2 \mathrm{~B}$. The multiple interventions (online supplemental file 2 ) were funded through CCBRT and made possible by the generous support of the European Union/ CBM (2010-2014), Australia CBM (2011-2015), Global Affairs Canada (20142019) and USAID/Nodafone (2015-2019). The authors acknowledge all partners and the implementations' funders listed in online supplemental file 1.

Contributors BSD, ZS and GM conceptualised the intervention and the paper. BSD is the technical advisor of the Tanzanian NGO CCBRT (www.ccbrt.or.tz), which supports strengthening of maternal and newborn healthcare in the DSM region, in collaboration with the regional health office. ZS and GM were in key leadership positions in the DSM region during implementation. BSD wrote the first draft and prepared the supplementary files. MM was responsible for data quality and preparation of the Excel data sheets. NH and BSD performed the data analysis and prepared figure 5. NM contributed to manuscript structure. All authors critically reviewed multiple versions of the manuscript and approved the final version. The authors alone are responsible for the views expressed in this article.
Funding This article is based on work done by the CCBRT maternal and newborn healthcare programme through the donors acknowledged and those listed in online supplemental file 1. The retrospective analysis is part of the PartoMa Project, which is funded by the Danida Fellowship Centre, Ministry of Foreign Affairs of Denmark (Danida project 18-08-KU).

Map disclaimer The depiction of boundaries on this map does not imply the expression of any opinion whatsoever on the part of BMJ (or any member of its group) concerning the legal status of any country, territory, jurisdiction or area or of its authorities. This map is provided without any warranty of any kind, either express or implied.

Competing interests None declared.

Patient consent for publication Not required.

Ethics approval Ethical approval to conduct this analysis was obtained from the Tanzanian National Institute for Medical Research (NIMR/HQ/R.8a/Vol.IX/3324).

Provenance and peer review Not commissioned; externally peer reviewed.

Data availability statement Data are stored at a repository at CCBRT and available on request.

Supplemental material This content has been supplied by the author(s). It has not been vetted by BMJ Publishing Group Limited (BMJ) and may not have been peer-reviewed. Any opinions or recommendations discussed are solely those of the author(s) and are not endorsed by BMJ. BMJ disclaims all liability and responsibility arising from any reliance placed on the content. Where the content includes any translated material, BMJ does not warrant the accuracy and reliability of the translations (including but not limited to local regulations, clinical guidelines, terminology, drug names and drug dosages), and is not responsible for any error and/or omissions arising from translation and adaptation or otherwise.

Open access This is an open access article distributed in accordance with the Creative Commons Attribution Non Commercial (CC BY-NC 4.0) license, which permits others to distribute, remix, adapt, build upon this work non-commercially, and license their derivative works on different terms, provided the original work is properly cited, appropriate credit is given, any changes made indicated, and the use is non-commercial. See: http://creativecommons.org/licenses/by-nc/4.0/.

\section{ORCID iDs}

Brenda Sequeira Dmello http://orcid.org/0000-0002-1550-248X

Natasha Housseine http://orcid.org/0000-0002-1849-7815

\section{REFERENCES}

1 Kruk ME, Kujawski S, Moyer CA, et al. Next generation maternal health: external shocks and health-system innovations. Lancet 2016;388:2296-306.

2 Sarah T, Donkin Angela BR. Creating healthy cities in Tanzania, 2016. Available: www.instituteofhealthequity.org [Accessed $15 \mathrm{Nov}$ 2019].

3 UNFPA, World Health Organization. Trends in maternal mortality 2000 to 2017: estimates by who, UNICEF, UNFPA, world bank group and the United nations population division ISBN 2019.

4 Hug L, Alexander M, You D, et al. National, regional, and global levels and trends in neonatal mortality between 1990 and 2017, with scenario-based projections to 2030: a systematic analysis. Lancet Glob Health 2019;7:e710-20.

5 Ministry of Health, Community Development, Gender Elderly and Children (MoHCDGEC) [Tanzania, Mainland], Ministry of Health (MoH) [Zanzibar], National Bureau of Statistics (NBS), Government Statistician (OCGS), 2016. Tanzania demographic and health survey and malaria indicator survey (TDHS-MIS) 2015-16 2016.

6 Save the Children Federation Inc. State of the worlds mothers 2015: the urban disadvantage, 2015.

7 Nyamtema AS, Urassa DP, Pembe AB, et al. Factors for change in maternal and perinatal audit systems in Dar es Salaam hospitals, Tanzania. BMC Pregnancy Childbirth 2010;10:29.

8 Nyamtema AS, Urassa DP, Massawe S, et al. Dar es Salaam perinatal care study: needs assessment for quality of care. East Afr $J$ Public Health 2008;5:17-21.

9 Nyamtema AS, Urassa DP, Massawe S, et al. Staffing needs for quality perinatal care in Tanzania. Afr J Reprod Health 2008;12:113-24.

10 Pembe AB, Paulo C, D'mello BS, et al. Maternal mortality at Muhimbili national hospital in Dar-es-Salaam, Tanzania in the year 2011. BMC Pregnancy Childbirth 2014;14:320. 
11 Nyamtema A, Rwamushaija E. CCBRT needs assessment for perinatal Care quality improvement for maternal and neonatal healthcare in Dar es Salaam region, 2009.

12 Pinnock H, Barwick M, Carpenter CR, et al. Standards for reporting implementation studies (STARI): explanation and elaboration document. BMJ Open 2017;7:e013318.

13 United Nations population projections. Dar ES Salaam, Tanzania Metro area population 1950-2020. Available: https://www. macrotrends.net/cities/22894/dar-es-salaam/population

14 MOHSW. Health sector strategic plan 2015 -2020. 53, 2015.

15 Shoo RS, Mboera LEG, Ndeki S. Stagnating maternal mortality in Tanzania: what went wrong and what can be done. Tanzan J Health Res 2017;19.

16 Rockers PC, Kruk ME, Laugesen MJ. Perceptions of the health system and public trust in government in low- and middle-income countries: evidence from the world health surveys. $J$ Health Polit Policy Law 2012;37:405-37.

17 White J, O‘Hanlon B, Grace Chee E. Tanzania private sector assessment, 2013. Available: www.shopsproject.org

18 Craig P, Dieppe P, Macintyre S, et al. Developing and evaluating complex interventions: the new medical Research Council guidance. Int J Nurs Stud 2013;50:587-92.

19 Ministry of Health (MoHCDGEC). The national road map strategic plan to improve reproductive, maternal, newborn, child \& adolescent health in tanzania (2016 - 2020), 2016. Available: www.moh.go.tz [Accessed 28 Mar 2020].

20 Columbia University (AMDD). Quality improvement for emergency obstetric care leadership manual, 2003.

21 Necochea E, Tripathi V, Kim Y-M, et al. Implementation of the Standards-Based management and recognition approach to quality improvement in maternal, newborn, and child health programs in low-resource countries. Int J Gynaecol Obstet 2015;130 Suppl 2:S17-24.

22 Kabo I, Otolorin E, Williams E, et al. Monitoring maternal and newborn health outcomes in Bauchi state, Nigeria: an evaluation of a standards-based quality improvement intervention. Int J Qual Health Care 2016;28:566-72.

23 Ayalew F, Eyassu G, Seyoum N, et al. Using a quality improvement model to enhance providers' performance in maternal and newborn health care: a post-only intervention and comparison design. BMC Pregnancy Childbirth 2017;17:115

24 R Core Team. R: a language and environment for statistical computing; R found. STAT. Comput. Vienna, Austria, 2019. Available: https://www.r-project.org/

25 McDougall L, Campbell OMR, Graham W. Maternal health an executive summary for the lancet's series. Lancet 2016.

26 Kruk ME, Pate M, Mullan Z. Introducing the Lancet global health Commission on high-quality health systems in the SDG era. Lancet Glob Health 2017:5:e480-1.

27 Kruk ME, Gage AD, Arsenault C. High-quality health systems in the Sustainable Development Goals era : time for a revolution. The Lancet Global Health Commission on High Quality Health Systems in the SDG Era. Lancet Glob Heal 2018;6:e1196-252.

28 Hanson C, Schellenberg J. Redesigning maternal health services: is centralisation the answer in low-resource settings? BMJ Glob Health 2019;4:e001488

29 Campbell OMR, Calvert C, Testa A, et al. The scale, scope, coverage, and capability of childbirth care. Lancet 2016;388:2193-208
30 Pollard S, Mathai M, Walker N. Estimating the impact of interventions on cause-specific maternal mortality: a Delphi approach. BMC Public Health 2013;13:S12.

31 Miller S, Abalos E, Chamillard M, et al. Beyond too little, too late and too much, too soon: a pathway towards evidence-based, respectful maternity care worldwide. Lancet 2016;388:2176-92.

32 McKay MM, Sensoy Bahar O, Ssewamala FM. Implementation science in global health settings: Collaborating with governmental \& community partners in Uganda. Psychiatry Res 2020;283:112585-48.

33 Tapela NM, Tshisimogo G, Shatera BP, et al. Integrating noncommunicable disease services into primary health care, Botswana. Bull World Health Organ 2019;97:142-53.

34 Peters DH, Adam T, Alonge O, et al. Implementation research: what it is and how to do it. BMJ 2013;347:f6753.

35 Pritchett L, Andrews M, Woolcock M. Escaping capability traps through problem driven iterative adaptation (PDIA). Center for International Development at Harvard University, 2017.

36 Singh K, Brodish P, Speizer I, et al. Can a quality improvement project impact maternal and child health outcomes at scale in northern Ghana? Health Res Policy Syst 2016;14:45.

37 Sensalire S, Isabirye P, Karamagi E, et al. Saving mothers, giving life approach for strengthening health systems to reduce maternal and newborn deaths in 7 scale-up districts in northern Uganda. Glob Health Sci Pract 2019;7:7:S168-87.

38 Kruk ME, Hermosilla S, Larson E, et al. Bypassing primary care clinics for childbirth: a cross-sectional study in the Pwani region, United Republic of Tanzania. Bull World Health Organ 2014:92:246-53.

39 Nyamtema AS, Mwakatundu N, Dominico S, et al. Enhancing maternal and perinatal health in Under-Served remote areas in sub-Saharan Africa: a Tanzanian model. PLoS One 2016;11:e0151419.

40 Bai X, Nath I, Capon A, et al. Health and wellbeing in the changing urban environment: complex challenges, scientific responses, and the way forward. Curr Opin Environ Sustain 2012;4:465-72.

41 Mayne J. Contribution analysis: coming of age? Evaluation 2012;18:270-80.

42 Delahais T, Toulemonde J. Applying contribution analysis: lessons from five years of practice. Evaluation 2012;18:281-93.

43 WHO. Standards for improving quality of maternal and newborn care in health facilities. WHO, 2019.

44 Leslie HH, Gage A, Nsona H, et al. Training and supervision did not meaningfully improve quality of care for pregnant women or sick children in sub-Saharan Africa. Health Aff 2016;35:1716-24.

45 Lewis TP, Roder-DeWan S, Malata A, et al. Clinical performance among recent graduates in nine low- and middle-income countries. Trop Med Int Health 2019;24:620-35.

46 Maaløe N, Housseine N, Bygbjerg IC, et al. Stillbirths and quality of care during labour at the low resource referral hospital of Zanzibar: a case-control study. BMC Pregnancy Childbirth 2016;16:351.

47 Maaløe N, Meguid T, Housseine N, et al. Local adaption of intrapartum clinical guidelines, United Republic of Tanzania. Bull World Health Organ 2019;97:365-70.

48 Penn-Kekana L, McPake B, Parkhurst J. Improving maternal health: getting what works to happen. Reprod Health Matters 2007;15:28-37.

49 Leslie HH, Hirschhorn LR, Marchant T, et al. Health systems thinking: a new generation of research to improve healthcare quality. PLoS Med 2018;15:e1002682-8. 